\title{
FAST RECONSTRUCTION ALGORITHMS FOR PERIODIC NONUNIFORM SAMPLING WITH APPLICATIONS TO TIME-INTERLEAVED ADCS
}

\author{
T. Strohmer* \\ University of California, Davis \\ Department of Mathematics \\ 1 Shield Ave \\ Davis, CA 95616-8633
}

\author{
J. Tanner ${ }^{\dagger}$ \\ University of Utah \\ Department of Mathematics \\ 155 South 1400 East, Room 233 \\ Salt Lake City, UT 84112-0090
}

\begin{abstract}
A bandlimited signal can be reconstructed from its periodic nonuniformly spaced samples provided the average sampling rate is at least the Nyquist rate. Unlike many previously published methods, the algorithm derived in this paper is designed that pays special attention to various practical constraints. In particular, we propose a fast and numerically robust reconstruction method which can utilize FIR filters with a small number of taps and requires only a modest amount of oversampling to achieve high accuracy. The efficiency and accuracy of the algorithm is obtained by fully exploiting the sampling structure combined with utilizing localized Fourier analysis. We discuss applications in time-interleaved analog-to-digital converters where nonuniform periodic sampling arises due to timing mismatches. Finally, numerical simulations demonstrate the performance of our algorithm.
\end{abstract}

Index Terms - Signal sampling, Signal reconstruction, Digital filters, Numerical analysis, Numerical stability.

\section{INTRODUCTION}

The ever-increasing desire for higher data rate and larger bandwidth of modern communication systems, such as ultrawideband communications, demands high-speed and high-resolution sampling systems. Furthermore, new methods in Sigma-Delta quantization require ADCs to operate at a substantial oversampling rate to achieve the required precision [1].

Current ADC technologies can on the one hand no longer meet these demands and on the other hand they cannot readily and cost-efficiently pushed further. A simple and appealing solution is to employ time-interleaved ADCs. A conventional ADC uniformly samples an analog signal and subsequently converts the discrete-time samples through quantization into a digital signal, which is then further processed. The key idea

\footnotetext{
${ }^{*}$ The first author acknowledges support from NSF DMS grant 0511461.

${ }^{\dagger}$ The second author would like to thank John E. and Marva M. Warnock for their generous support in the form of an endowed chair. Additional funding for this project came from NSF grants DMS-0135345 and DMS0403041 .
}

of a time-interleaved ADC is to send the analog input signal simultaneously through $N$ parallel ADCs, each operating at a rate $\frac{1}{T}$ and whose sampling instants are spaced such that the multiplexed output corresponds to a single ADC operating at rate $\frac{N}{T}$. Unfortunately, slight skews in the sample times of the individual ADCs destroy the overall uniform sampling pattern and result in a periodic nonuniform sampled output after multiplexing [2]. If the errors remain uncorrected one pays the price of a significant performance loss [2].

The correction of timing errors of time-interleaved ADCs comprises two steps. In the first step the timing offsets have to be estimated. Several algorithms have been published for this purpose, cf. [2] and the references therein. A particularly robust and computationally efficient method can be found in [3].

Here, we are interested in the second step, in which we have to eliminate the timing error effects, once the timing errors have been estimated. While this could be done by adjusting the sampling clock for each ADC, a better way is to apply interpolation to achieve the correct values at the ideal sampling instances [4], since the latter approach can be implemented using digital signal-processing circuits, which are portable and will benefit from evolving scaled CMOS technologies.

Thus, our goal in this paper is to design a method that can reconstruct a signal from such a sampling pattern. While literature offers a variety of methods for this purpose, e.g. [5, 6 , $7,8,9]$, we focus on developing a method that pays particular attention to practical constraints - such as the fact that only a finite number of samples is available - as well as numerical efficiency and robustness.

\section{UNIFORM (OVER)SAMPLING}

We briefly review some aspects of Shannon's sampling theorem from an angle that we be instrumental in introducing the proposed method for nonuniform periodic sampling. The famous Shannon sampling theorem says that a bandlimited signal can be recovered exactly from its equidistant samples via the cardinal series [10]. 
The uniform sampling operation induces periodization of a signal's Fourier transform. For $\sigma$-bandlimited signals this periodization can be removed if the sampling rate matches or exceeds the corresponding Nyquist rate, $T_{n y q}=1 / 2 \sigma$; the classical Shannon Sampling Theorem. Quantitatively,

$$
s(t) \equiv \sum_{k \in \mathbb{Z}} s(k T) \operatorname{sinc}_{\sigma}(t-k T), \quad \operatorname{sinc}_{\sigma}(t):=\frac{\sin (2 \pi \sigma t)}{2 \pi \sigma t}
$$

if and only if $T \leq T_{n y q}$ and $s \in B_{\sigma}$ where $B_{\sigma}$ is the space of $\sigma$-bandlimited signals ${ }^{1}$. For a multitude of practical reasons (such as robustness to noise and greater localization, which leads to smaller truncation error since in practice we are always dealing with finitely many samples) signals are traditionally oversampled, $T<T_{n y q}$, which allows for more general reconstruction pulses $\psi$. These pulses must satisfy the conditions $\psi(t) * \operatorname{sinc}_{\sigma}(t)=\operatorname{sinc}_{\sigma}(t)$ and $\psi \in B_{1 / 2 T}$, that is, $\psi$ 's Fourier transform being 1 for $|w| \leq \sigma$ and 0 for $|w| \geq 1 / 2 T$. For example, $\psi(\cdot)$, can be constructed to have root-exponential decay, $|\psi(t)| \leq C \cdot \eta_{\psi}^{-|t|^{1 / 2}}$ with $\eta_{\psi}>1$ ( $C$ is a constant) by smoothly connecting the region of 1 for $|w| \leq \sigma$ to 0 for $|w| \geq 1 / 2 T$ with dilation and translations of

$$
\rho(w):=\exp \left(-\gamma(1-w)^{-1} \exp (-1 / w)\right), \quad w \in(0,1),
$$

with $^{2} \gamma>0$, [11]. The root-exponential decay can be translated into finite-tap pulses with SNR gains (measured in $\mathrm{dB}$ ) proportional to the number of taps squared, cf. Sections 3 and 4.

The approximation of a signal via Shannon's Sampling Theorem from its finite number of samples, $2 L+1$, can be computed in $\mathcal{O}(L \log L)$ operations. Let $\left\{s_{\ell}\right\}_{|\ell| \leq L}$ be the $2 L+1$ uniform set of samples $s_{\ell}:=s(\ell T)$. Provided $T \leq$ $T_{n y q}$, an approximation of the signal can be recovered on the refined mesh with stepsize $T / p, p$ an integer, via frequency space filtering. Toward this end, let

$$
F_{j, k}:=\frac{T}{p \sqrt{2 \pi}} \exp \left(\frac{-2 \pi i p j k}{2 p L+1}\right)
$$

be the rectangular discrete Fourier transform of size $(2 p L+$ $1) \times(2 L+1)$ with $|j| \leq p L$ and $|k| \leq L$; and let

$$
F_{j, k}^{i n v}:=\frac{p \sqrt{2 \pi}}{T(2 p L+1)} \exp \left(\frac{2 \pi i j k}{2 p L+1}\right)
$$

be the square, $(2 p L+1) \times(2 p L+1)$, discrete inverse Fourier transform. Let $\Psi_{\ell}$ be the pulses Fourier transform on the mesh $p \ell / T(2 p L+1)$ for $|\ell| \leq p L$. Then, the approximation of the signal $s(\cdot)$ on the mesh $\ell T / p$ for $|\ell| \leq p L$ is given by

$$
\tilde{s}_{\psi}\left(\frac{k T}{p}\right):=F^{i n v} \Psi F S
$$

\footnotetext{
${ }^{1}$ Throughout this paper we will use lower case letters to designate signals in the time domain, and upper case for the Fourier transform of the same signal.

${ }^{2}$ Selecting $\gamma=e^{2} / 3$ gives good localization of $\psi(\cdot)$ for small $|t|$
}

with $S$ the vector with the samples entries $s_{\ell}$ and $\Psi$ the diagonal matrix of the filter's entries $\Psi_{\ell}$. Alternatively, an approximation of the signal can be computed on the translated mesh $t_{0}+\ell T / p$ by incorporating a modulation in the frequency domain,

$$
\tilde{s}_{\psi}\left(\frac{k T}{p}+t_{0}\right):=F^{i n v} M_{t_{0}} \Psi F S
$$

where $M_{t_{0}}$ is the diagonal matrix

$$
M_{t_{0}}:=\operatorname{diag}\left(\exp \left(\frac{-2 \pi i t_{0} \ell p}{(2 p L+1) T}\right)\right) \text { for }|\ell| \leq p L .
$$

The error in the approximation (5) is given by

$$
\left|s(t)-\tilde{s}_{\psi}(t)\right| \leq C \cdot \frac{p^{2} L}{\sigma^{2}}\|s\|_{L^{\infty}} B(L T-|t|)
$$

where $B(|t|)$ is a monotonically decreasing bound of the pulse $\psi(t)$. As stated before, root-exponential localization, $B(t) \leq$ $\eta_{\psi}^{-t^{\beta}}$ where $\eta_{\psi}>1$ and $\beta<1$, can be achieved by using Gevrey regular functions such as (2). The error in the approximation, (5) comes primarily from the finite number of samples, and to a lesser extent by replacing the Fourier Transform with the discrete Fourier transform. In fact, the errors introduced by the discrete Fourier transform are bounded by the finite sample error, [11].

\section{RECONSTRUCTION ALGORITHM FOR UNIFORM INTERLEAVED SAMPLING}

We consider the case of $N$ time-interleaved ADCs, the $n$-th ADC is supposed to sample the signal $s$ at instants $\{k T+$ $\left.\frac{(n-1) T}{N}\right\}_{k \in \mathbb{Z}}, n=1, \ldots, N$. Combining the outputs of the individual ADCs corresponds to a single ADC operating at sampling rate $\frac{N}{T}$. Due to the mentioned relative time skews of the individual sampling clocks, the $n$-th ADC actually samples

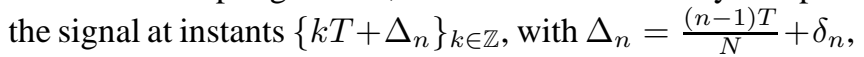
where the $\delta_{n}$ are the undesired timing offsets. Combining the $N$ individual sampling sets yields the periodic nonuniform sampling set $\left\{s\left(k T+\Delta_{n}\right)\right\}$, with $k \in \mathbb{Z}$ and $n=1, \ldots, N$. Of course, the combined average sampling rate is still $\frac{N}{T}$.

The following algorithm generalizes Shannon's Sampling Theorem to this sampling structure,

$$
s(t) \equiv \sum_{n=1}^{N} \sum_{k \in \mathbb{Z}} s\left(k T+\Delta_{n}\right) \psi_{n}\left(t-\left(k T+\Delta_{n}\right)\right) .
$$

We also present a fast discrete algorithm, similar to (5), for implementing the reconstruction.

Define the $n^{\text {th }}$ uniform set of samples as $\left\{s_{n, \ell}\right\}|\ell| \leq L$ where $s_{n, l}:=s\left(\ell T+\Delta_{n}\right)$, and let $S_{n}$ be the vector with entries $S_{n, \ell}:=s_{n, \ell}$ for $|\ell| \leq L$ and $n=1,2, \ldots, N$. Uniform sampling with offset $\Delta_{n}$ induces periodization of the $\Delta_{n}$ modulated signal's Fourier Transform. The sampling rate for any 
one of the uniform sampling sets being insufficient to recover the signal, that is $T>T_{n y q}$, corresponds to a periodization of the signal's Fourier Transform which overlaps with itself. However, if the effective sampling rate is sufficient, $T / N<T_{n y q}$, and provided the sampling translates $\Delta_{n}$ are distinct, the overlapping modulated periodization can be filtered to recover the original signal. The $N$ uniform sampling sets are used to recover the original signal's Fourier transform in intervals $I_{k}:=[\sigma+(-N+k-1) / T,-\sigma+k / T]$. This is achieved by solving for the filter coefficients $\left\{c_{k, n}\right\}_{n=1}^{N}$ that satisfy

$$
\sum_{n=1}^{N} c_{k, n} M_{\Delta_{n}} F S_{n}=F^{f u l l} S S_{p}
$$

where

$$
F_{j, k}^{f u l l}:=\frac{T}{p \sqrt{2 \pi}} \exp \left(\frac{-2 \pi i j k}{2 p L+1}\right)
$$

is the square discrete Fourier Transform and $S S_{p}$ is composed of the desired entries of $s(\cdot)$; that is, $s(\ell T / p)$ for $|\ell| \leq$ $p L$. This is possible provided the effective sampling rate is equal to or exceeds the Nyquist rate, $T / N \leq T_{n y q}$. The dependence of $s$ in equation (9) cancels, resulting in a small $N \times N$ system of equations for $\left\{c_{k, n}\right\}_{n=1}^{N}$. Let $R(k):=$ $\operatorname{diag}\left(\gamma_{1}(k) \gamma_{2}(k) \cdots \gamma_{N}(k)\right)$ with $\gamma_{n}(k):=\exp \left(2 \pi i \Delta_{n}(k-\right.$ $N-1) / T)$. Let $e_{N-k+1}$ be the usual unit direction with entries zero except for the $N-k+1^{t h}$ which is one, and let $c(k)$ be the column vector with entries $\left\{c_{k, n}\right\}_{n=1}^{N}$. The filter coefficients, $c(k)$, are determined by the system

$$
A R(k) c(k)=e_{N-k+1}, \quad A_{m, n}:=\exp \left(2 \pi i \Delta_{n} m / T\right) .
$$

We note that the matrix $A$ appears also in several other reconstruction algorithms, however it is important to note that it is utilized in a rather different way here.

Equipped with the filter coefficients $c_{k, n}$, the $I_{k}$-frequency band of $s(\cdot)$ can be recovered by multiplying the left hand side of (9) by a filter $\Phi_{k}(w)$ which is non-zero in $I_{k}$ and zero outside $I_{k}$. Repeating this process over a set of $I_{k_{j}}$ which cover $[-\sigma, \sigma]$ and using filters $\Phi_{k_{j}}$ which are a partition of unity,

$$
\sum_{j}^{\kappa} \Phi_{k_{j}}(w)=1 \quad \text { for } \quad|w| \leq \sigma
$$

allows for the recovery of $s(\cdot)$ over its entire bandwidth. The overall effect of this process upon a given sampling set $S_{n}$ is given by the filter

$$
\Psi_{n}(w):=\sum_{j=1}^{\kappa} c_{k_{j}, n} \Phi_{k_{j}}(w) .
$$

Finally, a simple calculation shows that an approximation to the signal $s(\cdot)$ on the mesh $\ell T / p$ for $|\ell| \leq p L$ is given by

$$
\tilde{s}_{\psi}\left(\frac{k T}{p}\right):=F^{i n v} \sum_{n=1}^{N} \Psi_{n} M_{\Delta_{n}} F S_{n},
$$

where $\Psi_{n}$ is the diagonal $2 p L+1$ square matrix with entries $\Psi_{n}(\ell, \ell):=\sum_{j=1}^{\kappa} c_{k_{j}, n} \Phi_{k_{j}}(\ell p / T(2 p L+1))$. An approximation of $s(\cdot)$ on a $t_{0}$-translated mesh can be obtained by inserting the modulation $M_{t_{0}}$, (7), between $F^{i n v}$ and the filters $\Psi_{n}$.

The above process is summarized in the following reconstruction algorithm for nonuniform periodic sampling.

\section{Algorithm 1:}

1. Select the mesh on which the approximation of the signal will be computed; that is, select the shift $t_{0}$ and integer refinement $p$ for the $2 p L+1$ length mesh $\left\{t_{0}+\right.$ $k T / p\}_{\mid k \leq p L}$.

2. From the signal $s(\cdot)$ 's bandwidth, $\sigma$, and the number of uniform sampling sets, $N$, select the $\kappa$ partition of unity, (12), functions $\Phi_{k_{j}}(\cdot)$.

3. From the sampling rate, $T$, and offsets of the uniform interleaved sampling sets, $\left\{\Delta_{n}\right\}_{n=1}^{N}$, solve (11) for the filter coefficients $\left\{c_{k_{j}, n}\right\}_{n=1}^{N}$ for $j=1,2, \ldots, \kappa$.

4. Combine the sets of $\Phi_{k_{j}}(\cdot)$ and $c_{k_{j}, n}$ from steps 1 and 2 to construct the filters $\Psi_{n}$ for $n=1,2, \ldots, N$ as in (13).

5. Form the samples $s_{\ell, n}$ into $N$ vectors $S_{n}$ with entries $S_{n}(\ell):=s_{\ell, n}$.

6. Apply the rectangular discrete Fourier $\operatorname{transform} F$, (3), to each sampling set $S_{n}$.

7. Apply modulation $M_{\Delta_{n}}$, (7), to the results from step 5, $M_{\Delta_{n}} F S_{n}$

8. Apply the filters $\Psi_{n}$, (13), to the results from step 6, $\Psi_{n} M_{\Delta_{n}} F S_{n}$.

9. Sum the results from step 7, multiply the result by $M_{t_{0}}$ (7), and apply the square discrete inverse Fourier Transform $F^{i n v}$, (4).

As in the case of (5), the accuracy of the approximation $\tilde{s}_{\psi}(\cdot)$ is determined by the localization properties of the pulses, $\psi_{n}(\cdot)$. The pulse localization follows directly from the smoothness of the partitions of unity, $\Phi_{k_{j}}(\cdot)$. A simple yet effective construction of the filter partitions is discussed in the next section.

\section{IMPLEMENTATION AND COMPLEXITY}

\subsection{A simple partition of unity}

A particularly simple construction of the partition of unity is to select the subset $k_{j}$ so that the intervals $I_{k}$ only overlap with their immediate neighbors, $I_{m} \bigcap I_{n}=\emptyset$ for $|m-n|>1$. To maintain a high degree of localization it is also advisable to have the overlap of the intervals as large as possible. These two natural conditions are satisfied with

$$
\kappa=\min \left(N,\left\lfloor\frac{N+1+T / T_{n y q}}{N+1-T / T_{n y q}}\right\rfloor\right)
$$


and

$$
k_{j}=\operatorname{round}\left(j \frac{N+1}{\kappa+1}-N\right) .
$$

The individual partitions, $\Phi_{k_{j}}(\cdot)$, can then be constructed to be 1 in regions where they do not overlap with their neighbors, and smoothly connecting for 0 for $w \notin I_{k}$ using dilation and translations of $\rho(\cdot),(2)$, and $1-\rho(\cdot)$. Explicit constructions for $\Phi_{k_{j}}(\cdot)$ are given in [11]. Note that the partition-filters are independent of the translates $\Delta_{n}$, depending only upon the signal's bandwidth, $\sigma$, and the number of uniform sampling sets, $N$.

The error for this reconstruction is

$$
\left|s(t)-\tilde{s}_{\psi}(t)\right| \leq C \cdot \frac{N^{2}}{\sigma}\|f\|_{L^{\infty}}\left\|A^{-1}\right\| \cdot B(L T-|t|)
$$

where $B(|t|)$ is a monotonically decreasing bound of the pulses $\psi_{n}$. The above construction with $\rho(\cdot)$ in (2) yields $B(t) \leq$ $\eta_{\psi}^{-|t|^{1 / 2}}$. For full details of the partition construction see [11].

\subsection{Computational complexity}

The direct implementation for uniform oversampling has a computational cost, limited by the FFT, of $\mathcal{O}(L \log L)$ where $L$ is the number of samples used in the reconstruction. The overall computational cost for Algorithm 1 is $\mathcal{O}(N L \log L)$ where $L$ is the total number of samples used in the approximation, cf. [11]. Thus, Algorithm 1 requires an additional factor of $N$ in the total computational cost, when compared to uniform sampling at the same effective sampling rate, and number of available samples.

\section{NUMERICAL SIMULATIONS}

We consider the case of 16 time-interleaved sampling sets and an overall oversampling rate of only $5 \%$. The input signals were generated as bandlimited AWGN, the timing errors $\delta_{n}$ are assumed to be Gaussian i.i.d. We truncate the pulses $\psi_{n}$ so that we utilize FIR pulses with a finite number taps, here the number of used taps ranges from 1 to 100 . For each fixed number of taps we compute the error between the exact sampling values of the input signal and those computed by Algorithm 1. We perform 2000 experiments and plot the average result in Figure 5. This experiment clearly demonstrates that the algorithm achieves high precision of the reconstructed signal with a modest number of taps and that the SNR increases exponentially (i.e., linearly on a dB-scale) with the squared number of taps, which is very desirable for an efficient DSP implementation of the proposed algorithm.

\section{REFERENCES}

[1] I. Daubechies and R. DeVore, "Approximating a bandlimited function using very coarsely quantized data: A family of stable sigma-delta modulators of arbitrary order," Annals of Mathematics, vol. 158, pp. 679-710, 2003.

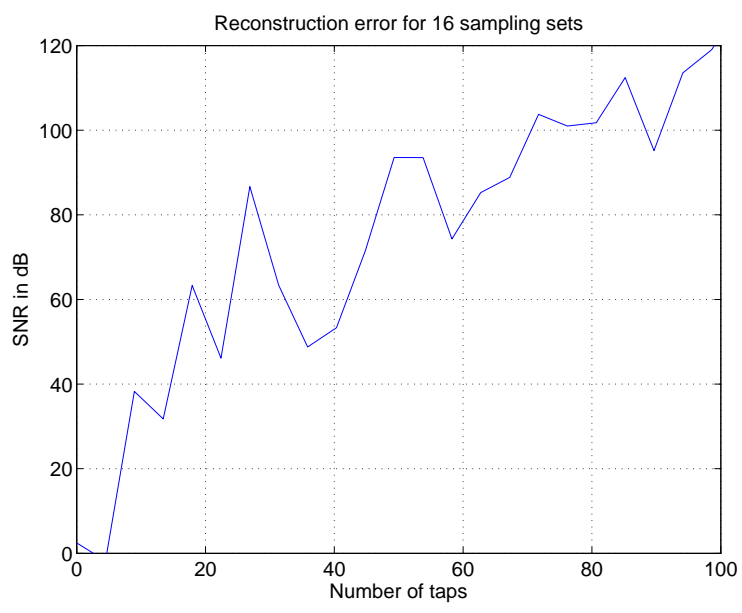

Fig. 1. The error in recovering the signal from 16 timeinterleaved sampling sets with $5 \%$ oversampling. The SNR increases exponentially (i.e., linearly on a dB-scale) with the squared number of taps of the pulses $\psi_{n}$, demonstrating that very high accuracy can be reached with short FIR pulses.

[2] C. Vogel and H. Johansson, "Time-interleaved analog-todigital converters: Status and future directions," in Proceedings of the 2006 IEEE International Symposium on Circuits and Systems (ISCAS 2006), 2006, pp. 3386-3389.

[3] J. Xu and T. Strohmer, "Efficient calibration of timeinterleaved ADCs via separable nonlinear least squares," 2006, preprint.

[4] W. Namgoong, "Finite-length synthesis filters for nonuniformly time-interleaved analog-to-digital converter," IEEE Trans. on Signal Processing, vol. 4, pp. 815-818, May 2002.

[5] A. Papoulis, Signal analysis, McGraw-Hill, New York, 1977.

[6] P.P. Vaidyanathan and V.C. Liu, "Efficient reconstruction of band-limited sequences from nonuniformly decimated versions by use of polyphase filter banks," IEEE Trans. Acoust. Speech Sig. Proc., vol. 38, pp. 1926-1936, 1990.

[7] H. Johansson and P. Löwenborg, "Reconstruction of nonuniformly sampled bandlimited signals by means of digital fractional delay filters," IEEE Trans. Acoust. Speech Sig. Proc., vol. 50, no. 11, pp. 2757-2767, 2002.

[8] F. Marvasti, Ed., Theory and Practice of Nonuniform Sampling, Kluwer/Plenum, 2001.

[9] R.S. Prendergast, B.C. Levy, and P.J. Hurst, "Reconstruction of bandlimited periodic nonuniformly sampled signals from multirate filter bank," IEEE Trans. Circ. Syst, to appear.

[10] A.I. Zayed, Advances in Shannon's Sampling Theory, CRC Press, Boca Raton, 1993.

[11] T. Strohmer and J. Tanner, "Fast reconstruction methods for bandlimited functions from periodic nonuniform sampling," SIAM J. Numer. Anal., vol. 44, no. 3, pp. 1073-1094, 2006. 